\title{
OSI Journal Editor Stakeholders Report
}

\author{
Kim Barrett; Patty Baskin; Susan Murray; Abel Packer; Margaret Winker
}

\section{Introduction}

The OSI journal editor stakeholder group was diverse in terms of geography and specialty field, with various backgrounds as either editors or in roles working closely with editors. Based on interests that aligned with the majority of the group at OSI2017, we decided to focus primarily on issues facing editors in the Global South. (While the use of the term "Global South" implies nonexistent homogeneity across the diverse countries, peoples, and cultures that comprise the Global South-the countries of Africa, Latin America, and developing Asia, including the Middle East - their editors and journals share issues across borders and continents, so the designation is useful.) OSI should convene a stakeholder group that explicitly comprises active editors at its next meeting to address their issues and concerns related to open access. For this summary, "editors" refers primarily to editors who make decisions on research manuscripts submitted to journals, although managing editors face many of these issues as well.

Regardless of where they are located globally, editors share a number of common issues and concerns. Responsibilities and expectations of all journal editors continue to increase as publishing becomes more complex. New guidelines and best practices are important for improving the quality of reported research, but they also require additional editors' time to ensure that journals, authors, and reviewers follow the guidelines. Editors' responsibilities traditionally include considering which manuscripts are appropriate for peer review, selecting reviewers, and carefully considering the revisions of authors and editing manuscripts accordingly. However, editors also must evaluate and handle the conflicts of interest of the authors, reviewers, and themselves; the authors' authorship criteria; the ethics of the research conducted; screening for plagiarism or self-plagiarism; and the possibility of research misconduct, including fabrication and falsification. They must require from authors complete reporting of research, and review (in addition to manuscripts) the research protocols, reporting guidelines, study registration, and sometimes raw data. Editors are expected to identify errors and authors' attempts to deceive, even as some authors pay third party organizations to circumvent peer review. ${ }^{1}$ Some researchers are paid thousands of dollars if they are able to get their research published, creating huge incentives that encourage deceiving editors. Each new way authors find to manipulate research, peer review, and publishing has meant that editors must find a way to identify problems and prevent problematic research or reporting from being published. If not, they risk having their journals called out in the international spotlight when articles must be retracted. Journals that are unable to afford the tools required to meet some standards risk being labeled as predatory. ${ }^{2}$ Insti-

(C) 2017 OSI2017 Journal Editors Stakeholder workgroup. This open access article is distributed under the Creative Commons Attribution 4.0 International License. This document reflects the combined input of the authors listed here (in alphabetical order by last name) as well as contributions from other OSI2017 delegates. The findings and recommendations expressed herein do not necessarily reflect the opinions of the individual authors listed here, nor their agencies, trustees, officers, or staff. 
tutions and funders have been slow to accept responsibility for the authors they support.

The members of the journal editor stakeholder group believe in the importance of open access. However, owners of journals that are open access may expect more efficiency from their editors because of resource constraints. Nearly all editors are expected to do more work than in past decades, often with fewer resources. Many editors are not paid and do their work as a passion and calling after their "day jobs" and academic responsibilities are over. Editors of open access journals that do not charge article processing charges (APCs), usually because their authors cannot afford them, may face even more substantial resource constraints. Some may face pressure from their journal owner that risks challenging editorial independence.

As stated in the Guardian, “....although digital technology and the internet have created a new terrain in which the ideals of open access have begun to germinate, they have yet to produce a cost-effective and reliable harvest of accessible knowledge." Without devoting more resources and/or technological efficiencies to help editors fulfill their obligations, the growth in journal requirements and editor responsibilities is not sustainable. The research community needs to develop better ways of motivating and enforcing the ethical behavior and appropriate research of its academics, rather than relying on editors as the final common pathway.

Editors of the Global North and South share the challenges of having to do more with the same or fewer resources, the need for screening tools to identify issues efficiently and accurately, and the need to be able to prioritize tasks. Editors in the North and South are also acutely aware of the need for academic institutions and funding organizations to share responsibility for promoting ethical behavior and the complete and reproducible reporting of research.

\section{Editors in the Global South}

Stakeholders across OSI tend to think of journals in terms of the high visibility titles often featured in the news media. Their editors are usually paid, may be full time, and have sufficient staff, resources, and influence for their journals to meet the requirements of indexers and achieve high(er) impact factors. However, editors of Global South and other small journals are often unpaid with few staff and little funding. ${ }^{3}$ Many have no publisher and therefore must assume responsibilities that the publisher normally would undertake. These editors often have little or no training, and no funds to pursue training. Many Global South journals are open access or free; only a few charge APCs because their authors cannot afford them. Many are supported by public funds, institutions, or societies. Unlike most journals in the Global North, Global South editors may play an informal role in mentoring authors and peer reviewers, since their regions lack the academic infrastructure and faculty to mentor authors and peer reviewers. Editors may provide substantive language editing because authors often lack language skills and do not have funding to hire professional editing services. These characteristics are not entirely unique to Global South journals; some small journals in the Global North share these challenges as well. However, the vast majority of these journals are from the Global South. 
In advance of OSI 2017, Margaret Winker conducted an April 2017 survey of members of the World Association of Medical Editors that highlighted several issues. First, while Global South editors reported improved internet and computer access (compared with a similar survey in 2013), access to research articles continues to be a challenge, with a third or more of Global South editors reporting that they did not have access to most of the research articles they required. Second, many journals in the Global South published their journals free or open access and did not charge APCs, instead depending on institutions or societies for support. Some published using CCBY, but many required the authors to transfer copyright, and some prohibited authors from depositing their final accepted manuscript or publishing their article in third party or institutional repositories. Third, many journals reported not having a data policy; of those that did, most required only that the author share data with the editor on request, not with researchers. Even so, some editors reported that some authors did not share the data on request or stated that they no longer had the data. Such data policies and experiences further challenge the reproducibility of the research literature and suggest the need for standards regarding data preservation and sharing. Fourth, journals in the Global South were consistently less likely than Global North journals to be indexed in Medline or Web of Science or to be found in PubMedCentral. When asked about barriers to indexing, some editors reported that they did not have the resources to meet the indexing requirements, did not understand the indexing requirements, or never heard back from the indexing organization. Finally, some editors were concerned about the impact of predatory or deceptive journals on their own journals, including being wrongly identified as a predatory journal. One journal wrongly identified as predatory lost so many author submissions that their indexing was revoked; another journal's name was deceptively appropriated by a predatory journal. These results highlight some of the challenges that editors face, particularly in the Global South.

\section{Journal Standards}

Journals around the world and the quality of the research they publish could benefit from clear, achievable, evidence-based journal standards. Such standards help editors focus their efforts on improving quality. Standards should not focus on mimicking the appearance of high cost, high impact factor journals; instead they should facilitate complete and transparent reporting, reproducibility, and discoverability of research. Furthermore, standards should not perpetuate and worsen the North/South journal divide by implementing standards that Global South journals are unable to afford. Standards should have few out-ofpocket financial requirements, or address how journals will meet them. For example, some standards have no direct costs (although they require person-hours to implement and maintain), such as the reporting standards for specific study designs, animal research, and the like, available on the EQUATOR Network. Other standards, such as DOI and archiving via sites like CLOCKSS (Closed Lots Of Copies Keeps Stuff Safe), have direct costs that journals must pay. While publishers and hosting organizations may have the opportunity and clout to negotiate reduced costs, individual journals do not have such opportunities. Journals unable to pay the costs of implementing standards should have the opportunity to do so at reduced cost. 
Furthermore, some standards, such as data preservation and sharing via an institutional repository, require institutional infrastructure and knowledge of issues, such as patient privacy concerns, that the author's institution may lack. Journals and authors should not be penalized for lack of infrastructure or expertise they have no control over. To help the research enterprise move forward in the Global South, affordable repositories should be encouraged. Use of options such as Figshare should be encouraged. Guidance on preserving research participant and patient privacy should be readily available to help all researchers achieve these goals.

Finally, while journals initially may achieve standards necessary to be indexed, some journals' adherence to standards declines over time, reducing their quality and jeopardizing their indexing. These examples of decline should be evaluated to identify reasons for decline, ways to prevent further decline, and methods to help journals continue to support quality publishing standards.

\section{Journal Indexing}

Small journals and journals in the Global South chronically face a lack of exposure to and discovery by readers. Lack of visibility can be related to Google search algorithms that require knowledge and investment to exploit ${ }^{4}$, as well as lack of journal indexing. Indexing is part of the virtuous cycle of better visibility attracting higher quality papers. While regional indexes exist in some parts of the Global South that help a subset of selected Global South journals gain visibility in their regions and internationally, such indexes may not give the journals the same degree of exposure as the traditional
Global North indexes. This sub-optimal international exposure not only limits the journal's growth but also prevents international readers from discovering research presented in journals that might be relevant to them. Limited research dissemination wastes research effort and funding and can also lead to needless research redundancy. Indexes that claim to be international should be truly international and make journals in the Global South easily accessible in the North, rather than invisible. If international indexes do not do so, alternatives must be found to ensure that search engine-based indexes such as Google Scholar identify individual articles and journals in the Global South and make them available via search results.

\section{Language Access}

Another barrier to "open" is language. If the open access movement focuses primarily on English language literature, many parts of the world will not benefit. Scholarly communication should encompass multilingualism in its standards, procedures and evaluation, in order to reflect the research context and purpose and to target the intended audience (including the public). Regardless of the discipline or type of research, most journal articles should be available in the language in which the research was conducted and for whom the research is intended. Journals may choose to publish in English in the hopes that they are more widely recognized, but that may make them less accessible to audiences with the most to gain. One solution is bilingual publication, but translations of research, for both researchers and the public, should take into account cultural and idiosyncratic contexts. Unfortunately, free electronic translation tools such as Google 
Translate are inadequate for translating the research literature. 5 Also, many journals and authors cannot afford professional translators. Therefore, until translation tools are improved, journals could ask authors to provide at least a translated abstract (after peer review and revision) to enable their research to be identified in at least the languages in which they have fluency. The journal may wish to add a disclaimer indicating that the translation was provided by the author.

\section{Importance of Journals to the Research Culture}

Editors don't just select articles for publication; they help develop academic scholarship by working with authors, reviewers, and editorial boards. The research culture includes researchers conducting peer review and serving on editorial boards. Editors help researchers acquire a more detailed understanding of academic publishing and the process of editorial evaluation and standards, including the issues that arise such as authorship, conflicts of interest, and research conduct and reporting. Institutions should encourage publication in their country's journals and provide academic recognition for the services that reviewers and editorial boards provide.

\section{Impact Factor}

Impact factor, and the emphasis placed on publishing in high impact factor journals, discourages Global South researchers from publishing in journals in their own countries. These policies pose several challenges for Global South journals and the recognition and progress of research regarding local problems or of local interest. First, because the impact factor relies on indexing in Global North indexes, Global South journals are at a disadvantage when competing on the basis of impact factor. Second, Global South journals are more difficult to find in Global-North dominated indexes and in search engines that prioritize search results based on web traffic. As a result, Global South journals may be less likely to garner citations for their articles, thereby undermining the impact factor. Third, and most importantly, impact factor is a poor substitute for measuring the impact of research, as set out by the Declaration on Research Assessment (DORA) and other initiatives. Promotion and tenure committees that prioritize impact factor over more accurate measures of articlelevel impact further undermine the journals of their own regions, and thereby the research culture. Their incentives promote research being removed from regions where it could have the most impact on cultural, social and economic development, especially with regards to health, environmental protection, and public policy.

As Vint Cerf remarked in his keynote address, changing behaviors requires examining incentives. Funding agencies and institutional promotion and tenure committees should use metrics other than impact factor to evaluate the work of researchers. They should recognize the value of publishing research in local journals, as well as publishing in the language of the research for those who would benefit from access to it. There are several concrete approaches funding agencies and institutional promotion and tenure committees could adopt to promote these alternative metrics. For example, agencies could use the approach of RCUK/MRC: evaluating the impact of research in the area where research is conducted, e.g., through influencing guidelines and/or policy. They could also use article metrics to evaluate the impact of research 
without resorting to the impact factor. Finally, they could adopt the suggestion by Keith Yamamoto that researchers be evaluated on their most impactful 5 or 10 works, be they research articles, data, or even code, without regard to impact factor or even the journal in which they were published.

\section{Importance of Mentorship}

Mentors (local or otherwise) are an important resource for authors and editors. ${ }^{6}$ However, rather than mentors from the Global North informing authors and editors of the Global South how they should best execute their work, mentoring should take into account local practices and approaches. Just as the most effective solutions in Manhattan may not apply in Mississippi, issues should be assessed and solutions developed using local perspectives. Furthermore, any behavior change requires buy-in from stakeholders-including in the Global South. Local incentives must be evaluated to determine how to change behaviors most effectively.

Networking and collaboration depend on identifying researchers and others working in related areas. However, unlike the Global North, the Global South has fewer such networks. Increasing the participation of Global South researchers in these networks and the promotion of Global South networks of researchers could facilitate collaboration in the same continent, country, or language. Global South research networks could aid the role of mentors and help identify appropriate peer reviewers for completed research.

Not all efforts need to be local. Professional specialty societies in the Global
North and South could partner to share information and experiences. For example, a US ophthalmological society could pair with a comparable ophthalmological society in Malawi. The partnership could discuss how to further academic activities in both locations, including cross-appointed editorial board members and peer reviewers, joint conferences, etc., promoting learning for both North and South.

\section{Learning from the "South"}

Sharing information is a two-way street. The Global South traditionally has taken a different approach to scholarly publishing, based more on necessity than earnings. Therefore, rather than developing expensive solutions that support publisher profit, the lower expense solutions developed in the Global South may help the research enterprise in general reduce the cost of publishing, thereby making universal open access more feasible. Some Global South non-profit indexes also provide "metapublisher" services to journals they accept to the platforms. For example, SciELO Latin America provides a common publishing platform and solutions to journals, using a version of the free or low cost open source Open Journal Systems (OJS), developed by the Public Knowledge Project (PKP), which was modified in-house. SciELO is developing an editing tool for authors to tag their own XML and generate a PDF. Even if a review of the final product were required to ensure accurate tagging, such a tool could help distribute the work of article markup and reduce the expense of publishing. African Journals Online (AJOL) - also based on OJS and amended in-house-similarly provides free aggregator hosting of journals' content, the option for free hosted online publishing, and free 
DOIs to its approved partner journals.

These and similar organizations not only support and index, but will also evaluate journals, providing education for editors to help them achieve higher standards. Using solutions from around the world will help editors, researchers, and those who benefit from research.

\section{“Open” Questions}

Several "open" questions remain regarding the future of editors in general, as well as the future of Global South journals, editors, academic institutions, and the research culture. These include:

1) Editors increasingly must identify research misconduct and prevent unethical behaviors on the part of authors and reviewers. How can responsibility for enforcing ethical research standards be shared more equitably with institutions and funders?

2) How can those in the Global South publish open access journals that meet quality standards when their authors cannot afford APCs? Who will pay for publication-government, institutions, funders? How can sustainability be preserved? How can conflicts of interest be avoided?

3) What are the most effective ways to change academic culture to value openness and to value publishing regionally, and in the research language?

4) Can automated translating tools be improved sufficiently to provide reliable translations of research (particularly medical research)?

\section{Actions}

\section{Standards:}

- Establish (with global representation) clear, achievable, evidencebased journal standards focused on improving the quality, transparency, and reproducibility of research, rather than the appearance of the journal. Standards should have few outof-pocket financial requirements and means for journals to pay for them should be addressed.

- Contact CrossRef and CLOCKSS regarding how to achieve (markedly) reduced costs for Global South and other small under-resourced journals.

- Develop (with global representation) data policy standards regarding authors' retaining and sharing data.

- Identify free or nearly free data repositories such as Figshare for author and editor reference.

- Develop (with global representation) standards for data privacy for Global South authors, institutions, and editors to use.

- Develop (with global representation) approaches for Global South institutions to develop institutional repositories - funding and best practices.

- Study why some journals may cease to adhere to standards and determine ways to prevent declining standards.

\section{Indexing:}

- Catalog requirements of major indexes for editors to easily reference; synthesize requirements into standards to improve likelihood of indexing; identify issues with Global South journal practices that impede 
indexing, and causes and ways to alter their practices.

- Identify liaisons at major indexing organizations to turn to when editors have questions.

- Until truly global indexing is available, strengthen regional journal indexes that national research evaluation systems, institutions and researchers (including systematic reviewers) can use to ensure that they are capturing all relevant research.

- Evaluate standards of "international" indexes to determine why Global South journals are preferentially not indexed.

- Approach indexing organizations regarding requirements that may not be essential and inequality practices that may introduce bias against Global South journals.

- Approach Google Scholar re: increasing the likelihood that Global South journals and articles will appear in search results.

\section{Language Access:}

- Identify (with global representation) ways to encourage journals to publish in the main language of the country --with English abstracts provided by the author if the journal cannot afford professional translation.

- Convey (with global representation) the importance of publishing in the country's language to academic institutions within the country.

- Convey to Google (with global representation) the importance of improving automated translations of research (particularly medical research) to at least improve the first pass of research translation before professional translators or authors refine translations.

\section{Importance of Journals to the Research Culture:}

- Convey to academic institutions and funders the importance of journal editors to the culture of academic scholarship.

- Encourage institutions to recognize the services that peer reviewers and editorial boards provide as important academic achievements.

\section{Impact Factor:}

- Convey to Global South academic institutions and funding organizations the problems that the use of impact factor and publication in Global North journals as criteria for research impact create for Global South journals and the fostering of academic culture in the Global South; explain the limitations of impact factor and the alternative means of judging impact set out by DORA and implemented by some funding organizations such RCUK/MRC.

- Examine incentives for Global South researchers and how incentives might be changed to promote open publishing and publishing in Global South journals.

\section{Importance of Mentorship:}

- Examine with potential funders ways in which a Global South network might be developed, incorporating existing standards such as ORCID.

- Contact scholarly societies to determine feasibility of new programs pairing specialty societies in the Global North and South. 
Learning from the "South":

- Create a clearinghouse for ways in which journals, publishers, and indexers in the Global South and North are improving quality, implementing standards, streamlining publishing, evaluating journals, or otherwise improving the publishing process. The clearinghouse should be available for researchers to evaluate the efficacy of particular approaches for different regions of the world.

\section{“Open" Questions:}

- Develop (with global representation) best practices for journals based on their funding model, including those funded by government, institutions, and other funders, to preserve editorial freedom and prevent conflicts of interest.

- Involve stakeholders in various regions in discussions about how to change academic culture to value openness and to value publishing regionally in the research language.

- Involve stakeholders to identify ways in which institutions and funders can incentivize ethical research and detect and prevent research misconduct.

Disclaimer: The ideas presented herein do not necessarily represent those of the Council of Science Editors or World Association of Medical Editors.

\section{References}

1. Best Practices for Peer Reviewer Selection and Contact to Prevent Peer Review Manipulation by Authors. World Association of Medical Editors. April 6, 2015. http://www.wame.org/policy-statements \#Best $\% 20$ Practices $\% 20$ for $\% 20 \operatorname{Peer} \% 20$ Reviewer $\% 20$ Selection

2. Laine C, Winker M. Identifying Predatory or Pseudo-Journals. World Association of Medical Editors. February 18, 2017. http://www.wame.org/identifying-predatory-or-pseudojournals

3. Curry S. It's time for academics to take back control of research journals. The Guardian. May 25, 2017. https://www.theguardian.com/higher-education-network/2017/may/25/its-time-for-academics-to-take-back-control-of-research-journals

4. Marusic A, Marusic M. Small scientific journals from small countries: breaking from a vicious circle of inadequacy. Croatian Medical Journal 1999; 40(4): 508-14.

5. Anderson K. Detours and Diversions-Do Open Access Publishers Face New Barriers?Scholarly Kitchen blog. May 31, 2017. https://scholarlykitchen.sspnet.org/2017/05/31/detours-and-diversions-do-open-access-publishers-face-new-barriers/ 
6. Balk EM, Chung M, Chen ML, et al. Assessing the accuracy of Google Translate to allow data extraction from trials published in non-English languages. Agency for Healthcare Research and Quality (US); 2-13 Jan. Report No.: 12(13)-EHC145-EF.

7. Sambunjak D. Understanding wider environmental influences on mentoring: Towards an ecological model of mentoring in academic medicine. Acta Med Acad. 2015;44(1):47-57. doi: 10.5644/ama2006-124.126.

\section{Journal Editors Stakeholder Group:}

Kim Barrett, Editor-in-Chief, The Journal of Physiology; Past-President of the American Physiological Society

Patty Baskin, Executive Editor of the Neurology Journals; President, Council of Science Editors

Susan Murray, Managing Director of AJOL

Abel Packer, Co-founder and current Coordinator, SciELO

Margaret Winker, World Association of Medical Editors (primary author) 